Теорія Ймовір. та Матем. Статист. Вип. 74, 2006
Theor. Probability and Math. Statist.

No. 74, 2007, Pages 25-28

S 0094-9000(07)00694-1

Article electronically published on June 25, 2007

\title{
THE LIMIT VALUE OF THE PRICE OF A EUROPEAN CALL OPTION IN THE BINOMIAL MODEL
}

UDC 519.21

\author{
S. O. GOROVYI
}

\begin{abstract}
We find the limit value of the price of a European call option in the binomial model if the strike price does not change but the number of steps in the model tends to infinity. We assume that the market is arbitrage free.
\end{abstract}

\section{INTRODUCTION}

The Cox-Ross-Rubinstein binomial model describing the dynamics of the stock price is sometimes used to analyze financial markets. This model involves a bond whose interest rate $r$ is constant and only one risky asset whose dynamics for all $t \geq 0$ is given by

$$
S(t+1)= \begin{cases}S(t)(1+b), & \text { with probability } p, \\ S(t)(1+a), & \text { with probability } q=1-p .\end{cases}
$$

It is also assumed that $0<1+a<1+r<1+b$. Since the market is arbitrage free, a martingale measure $\left(p^{*}, q^{*}\right)$ exists and satisfies the equality

$$
p^{*}(1+b)+q^{*}(1+a)=1+r .
$$

A financial derivative is called a European call option if it gives a right (not an obligation) to its owner to purchase one share of a stock from the seller for a strike price $E$. The owner of a call option may exercise it at an expiration date $T$ fixed at the moment when he purchased the option. The value of an option at the expiration time $T$ is $(S(T)-E)^{+}$.

\section{MAIN RESUlT}

Theorem 2.1. Consider the binomial model of the financial market. The limit value of the price of a European call option as $T \rightarrow \infty$ and if all other parameters of the model are constant is given by

$$
S= \begin{cases}S(0), & \lambda<\tilde{p} \\ \frac{S(0)}{2}, & \lambda=\tilde{p} \\ 0, & \lambda>\tilde{p}\end{cases}
$$

where

$$
\lambda=\frac{-\ln (1+a)}{\ln (1+b)-\ln (1+a)}, \quad \tilde{p}=\frac{p^{*}(1+b)}{1+r} .
$$

2000 Mathematics Subject Classification. Primary 91B28; Secondary 91B62.

Key words and phrases. The price of an option, European option, binomial model. 
Proof. First we use the Cox-Ross-Rubinstein formula for the price of a European call option for a model with $n$ steps [1]:

$$
S_{n}=\frac{1}{(1+r)^{n}} \sum_{k=0}^{n}\left(\begin{array}{l}
n \\
k
\end{array}\right)\left(p^{*}\right)^{k}\left(q^{*}\right)^{n-k}\left(S(0)(1+b)^{k}(1+a)^{n-k}-E\right)^{+} .
$$

If $S(0)(1+b)^{k}(1+a)^{n-k}>E$, that is,

$$
k>\frac{\ln E-\ln S(0)-n \ln (1+a)}{\ln (1+b)-\ln (1+a)},
$$

then the corresponding term on the right hand side of (1) is nonzero.

1. First we consider the case of $\ln (1+a)>0$. Then inequality (2) holds for all $k \geq 0$ if $n$ is sufficiently large. Thus one can rewrite equality (11) as

$$
\begin{aligned}
S_{n} & =\frac{1}{(1+r)^{n}} \sum_{k=0}^{n}\left(\begin{array}{l}
n \\
k
\end{array}\right)\left(p^{*}\right)^{k}\left(q^{*}\right)^{n-k}\left(S(0)(1+b)^{k}(1+a)^{n-k}-E\right) \\
& =\frac{S(0)}{(1+r)^{n}} \sum_{k=0}^{n}\left(\begin{array}{l}
n \\
k
\end{array}\right)\left(p^{*}(1+b)\right)^{k}\left(q^{*}(1+a)\right)^{n-k}-\frac{E}{(1+r)^{n}} \\
& =S(0)\left(\frac{p^{*}(1+b)+q^{*}(1+a)}{1+r}\right)^{n}-\frac{E}{(1+r)^{n}}=S(0)-\frac{E}{(1+r)^{n}} .
\end{aligned}
$$

The latter expression obviously tends to $S(0)$ as $n \rightarrow \infty$. It is easy to check that $\lambda<0<\tilde{p}$ for this case.

2. Now we consider the case of $\ln (1+a)=0$, that is, $a=0$. In this case, the sum on the right hand side of (11) contains the terms whose indices $k$ satisfy inequality (2) for sufficiently large $n$. The right hand side of inequality (2) is a constant that does not depend on $n$. Denote the minimal integer number that does not exceed this constant by $k_{0}$. Then the sum in (11) runs over those indices $k$ that are greater than or equal to $k_{0}$. Consider the terms with $k<k_{0}$. We show that every term with $k<k_{0}$ does not change the limit. Indeed,

$$
\begin{aligned}
\frac{1}{(1+r)^{n}}\left(\begin{array}{l}
n \\
k
\end{array}\right)\left(p^{*}\right)^{k}\left(q^{*}\right)^{n-k}\left(S(0)(1+b)^{k}-E\right)^{+} \\
\leq \frac{1}{(1+r)^{n}}\left(\begin{array}{l}
n \\
k
\end{array}\right)\left(p^{*}\right)^{k}\left(q^{*}\right)^{n-k}\left(S(0)(1+b)^{k}-E\right) \\
\leq \frac{S(0)}{(1+r)^{n}} \frac{n \cdots(n-k+1)}{k !}\left(p^{*}\right)^{k}\left(q^{*}\right)^{n-k}(1+b)^{k} \\
\leq \frac{S(0)}{k !}\left(\frac{n p^{*}(1+b)}{q^{*}}\right)^{k}\left(\frac{q^{*}}{1+r}\right)^{n}
\end{aligned}
$$

where $q<1+r$. Thus the right hand side of the latter inequality approaches 0 . We see that these $k_{0}$ terms do not influence the limit value of the sum as the number of steps tends to infinity, so the limit is the same as in the preceding case, namely $S(0)$. It is also easy to check that $\lambda=0<\tilde{p}$ in this case.

3. Finally we consider the case of $\ln (1+a)<0$. Then

$$
k_{n}=1+\left[\frac{\ln E-\ln S(0)-n \ln (1+a)}{\ln (1+b)-\ln (1+a)}\right]
$$


has the same order of growth as $n$. We rewrite the sum (11) as

$$
\begin{aligned}
S_{n}= & \frac{1}{(1+r)^{n}} \sum_{k=k_{n}}^{n}\left(\begin{array}{l}
n \\
k
\end{array}\right)\left(p^{*}\right)^{k}\left(q^{*}\right)^{n-k}\left(S(0)(1+b)^{k}(1+a)^{n-k}-E\right) \\
= & S(0) \sum_{k=k_{n}}^{n}\left(\begin{array}{l}
n \\
k
\end{array}\right)\left(\frac{p^{*}(1+b)}{1+r}\right)^{k}\left(\frac{q^{*}(1+a)}{1+r}\right)^{n-k} \\
& -E \frac{1}{(1+r)^{n}} \sum_{k=k_{n}}^{n}\left(\begin{array}{l}
n \\
k
\end{array}\right)\left(p^{*}\right)^{k}\left(q^{*}\right)^{n-k} \\
= & S(0) \sum_{k=k_{n}}^{n}\left(\begin{array}{l}
n \\
k
\end{array}\right)\left(\frac{p^{*}(1+b)}{1+r}\right)^{k}\left(\frac{q^{*}(1+a)}{1+r}\right)^{n-k}-E f(n) .
\end{aligned}
$$

Since

$$
0 \leq f(n) \leq \frac{1}{(1+r)^{n}} \sum_{k=0}^{n}\left(\begin{array}{l}
n \\
k
\end{array}\right)\left(p^{*}\right)^{k}\left(q^{*}\right)^{n-k}=\frac{1}{(1+r)^{n}},
$$

we obtain $f(n) \rightarrow 0$ as $n \rightarrow \infty$. Therefore

$$
\lim _{n \rightarrow \infty} S_{n}=S(0) \lim _{n \rightarrow \infty} \sum_{k=k_{n}}^{n}\left(\begin{array}{l}
n \\
k
\end{array}\right)\left(\frac{p^{*}(1+b)}{1+r}\right)^{k}\left(\frac{q^{*}(1+a)}{1+r}\right)^{n-k} .
$$

Put

$$
\tilde{p}=\frac{p^{*}(1+b)}{1+r}, \quad \tilde{q}=\frac{q^{*}(1+a)}{1+r} .
$$

Then $\tilde{p} \geq 0$ and $\tilde{q} \geq 0$. Moreover $\tilde{p}+\tilde{q}=1$ in view of the assumption that there is no arbitrage strategy. This implies that the prelimit expression on the right hand side of (6) is the probability that the number of successes $\xi_{n}$ in $n$ Bernoulli trials is not less than $k_{n}$ if the probability of the success in a single Bernoulli trial is $\tilde{p}$ and the probability of the failure is $\tilde{q}$. It is well known that the normalized random variable

$$
\eta_{n}=\frac{\xi_{n}-n \tilde{p}}{\sqrt{n \tilde{p} \tilde{q}}}
$$

weakly converges to a standard Gaussian random variable.

We distinguish between the following three cases.

1) The case of $\lambda<\tilde{p}$. Then for any real number $x$, one can find an integer number $n_{x}$ such that

$$
\frac{k_{n}-n \tilde{p}}{\sqrt{n \tilde{p} \tilde{q}}}<x
$$

for all $n>n_{x}$, that is,

$$
\mathrm{P}\left\{\xi_{n} \geq k_{n}\right\}=\mathrm{P}\left\{\frac{\xi_{n}-n \tilde{p}}{\sqrt{n \tilde{p} \tilde{q}}} \geq \frac{k_{n}-n \tilde{p}}{\sqrt{n \tilde{p} \tilde{q}}}\right\} \geq \mathrm{P}\left\{\frac{\xi_{n}-n \tilde{p}}{\sqrt{n \tilde{p} \tilde{q}}} \geq x\right\}=\mathrm{P}\left\{\eta_{n} \geq x\right\} .
$$

Since $\mathrm{P}\left\{\eta_{n} \geq x\right\} \rightarrow 1-\Phi(x)$ where $\Phi(x)$ denotes the standard Gaussian distribution function, we obtain

$$
1 \geq \lim _{n \rightarrow \infty} \mathrm{P}\left\{\xi_{n} \geq k_{n}\right\} \geq 1-\Phi(x) \quad \text { for all } x \in \mathbb{R} .
$$

Therefore $\lim _{n \rightarrow \infty} \mathrm{P}\left\{\xi_{n} \geq k_{n}\right\}=1$ and relation (6) implies that the limit value of the price of a European call option equals the initial stock price.

2) The case of $\lambda=\tilde{p}$. Then

$$
x_{n}=\frac{k_{n}-n \tilde{p}}{\sqrt{n \tilde{p} \tilde{q}}} \rightarrow 0
$$


and $x_{n}<x$ for any given $x>0$ and for all sufficiently large $n$. Similarly, $x_{n}>x$ for a given $x<0$ and for all sufficiently large $n$. This implies that

$$
\lim _{n \rightarrow \infty} \mathrm{P}\left\{\xi_{n} \geq k_{n}\right\}=1-\Phi(0)=\frac{1}{2},
$$

that is, the limit value of the price of a European call option equals half of the initial stock price.

3) The case of $\lambda>\tilde{p}$. For a given $x$ one can find $n_{x}$ such that

$$
\frac{k_{n}-n \tilde{p}}{\sqrt{n \tilde{p} \tilde{q}}}>x
$$

for all $n>n_{x}$, that is,

$$
\mathrm{P}\left\{\xi_{n} \geq k_{n}\right\}=\mathrm{P}\left\{\frac{\xi_{n}-n \tilde{p}}{\sqrt{n \tilde{p} \tilde{q}}} \geq \frac{k_{n}-n \tilde{p}}{\sqrt{n \tilde{p} \tilde{q}}}\right\} \leq \mathrm{P}\left\{\frac{\xi_{n}-n \tilde{p}}{\sqrt{n \tilde{p} \tilde{q}}} \geq x\right\}=\mathrm{P}\left\{\eta_{n} \geq x\right\} .
$$

Since $\mathrm{P}\left\{\eta_{n}>x\right\} \rightarrow 1-\Phi(x)$ where $\Phi(x)$ is the standard Gaussian distribution function, we get

$$
0 \leq \lim _{n \rightarrow \infty} \mathrm{P}\left\{\xi_{n} \geq k_{n}\right\} \leq 1-\Phi(x) \text { for all } x \in \mathbb{R} .
$$

Therefore $\lim _{n \rightarrow \infty} P\left\{\xi_{n} \geq k_{n}\right\}=0$ and relation (6) implies that the limit value of the price of a European call option is equal to 0 .

This completes the proof of the theorem.

\section{Concluding Remarks}

The limit value of the price of a European call option is found in this paper as the number of steps in the binomial model increases to infinity. It is interesting that this limit does not depend on the strike price.

Among possible extensions of this result we mention a problem on the rate of convergence of the price of a European call option to the limit. Similar questions arise in analogous problems for the polynomial model, that is, for the case where $S(t+1)=S(t)\left(1+a_{k}\right)$ with probability $p_{k}, 1 \leq k \leq m$, and for the case where the strike price depends on the expiration date.

\section{BIBLIOGRAPHY}

1. Robert J. Elliott and P. Ekkehard Kopp, Mathematics of Financial Markets, Springer-Verlag, New York, 1999. MR1674047(2000b:91067)

Department of Probability Theory and Mathematical Statistics, Faculty for Mathematics and Mechanics, National Taras Shevchenko University, Glushkov Avenue, 6, Kyiv, 03127, UKRAINE

E-mail address: sg@univ.kiev.ua

Received 19/JAN/2005

Translated by OLEG KLESOV 